\title{
Síntese dos resultados dos inquéritos nacionais
}

\author{
Summary of results from the national surveys
}

\author{
Afonso Dinis Costa Passos*e Antônio Carlos Silveira**
}

\begin{abstract}
RESUMO
Aqui se busca correlacionar os resultados dos grandes inquéritos nacionais realizados no final da década de 1970 e início da década de 1980 (entomológico, sorológico e eletrocardiográfico) que serviram de base para orientar as ações de controle da doença de Chagas no país. Verificouse uma maior proporção de infectados nas áreas correspondentes àquela de distribuição de Triatoma infestans, a espécie reconhecidamente com maior capacidade vetorial entre as cinco identificadas como as mais importantes no Brasil à época. Achado similar foi observado para a área de dispersão de Triatoma sordida, pela coincidência existente com grande parte daquela de distribuição de T. infestans, especialmente na região central do país de onde é nativa, e não pela sua relevância na transmissão. No semiárido do nordeste do país, centro de endemismo de Triatoma brasiliensis e de Triatoma pseudomaculata, as taxas de soro-prevalência de infecção humana foram bastante menores, ainda que se possa atribuir a ambos vetores importância na manutenção da endemia chagásica na região. Para Panstrongylus megistus ocorreram variações de acordo com as suas características de maior ou menor domiciliação. Sempre que domiciliado pode-se comprovar ter papel relevante na transmissão domiciliar de Trypanosoma cruzi, como no litoral úmido do nordeste, onde em alguns casos era vetor exclusivo, como no Recôncavo Baiano. A partir dos dados do inquérito de soroprevalência foi realizado estudo eletrocardiográfico em onze estados, o qual mostrou variações regionais evidentes nas manifestações clínicas observadas.
\end{abstract}

Palavras-chaves: Doença de Chagas. Inquéritos epidemiológicos.

\begin{abstract}
This article aims to correlate the main results of three large national surveys on Chagas disease (entomologic, seroprevalence and electrocardiographic) carried out in Brazil from late 1970's to early 1980's, which served as baseline for definition of the control measures adopted in the country. The proportion of infected people was much higher in areas where Triatoma infestans, the most efficient vector of Chagas disease among the five principal species involved in transmission at that time, was predominant. Similar result was observed in places where Triatoma sordida was dispersed, mainly in the country's central region, which corresponds to its native area. This finding is due to the coincidence observed in the geographic distribution of both vectors, since T. sordida is not considered to play an important role in transmission. In the Northeastern semi-arid, endemic area for Triatoma brasiliensis and Triatoma pseudomaculata, rates of human infection were much lower, although both vectors may have some relevance in the maintenance of the disease. As for areas with Panstrongylus megistus, human infection varied according to the levels of domiciliation. Whenever domiciled, like in the humid northeastern coastal area, its involvement in transmission can be clearly demonstrated. In some parts of Bahia State it represented the exclusive vector of the disease. Based upon the results of the seroprevalence survey an electrocardiographic study was carried out in 11 Brazilian states, which showed marked differences in the presence of cardiac alterations when comparing different areas of the country.
\end{abstract}

Key-words: Chagas disease. Epidemiological surveys.

Entre a segunda metade dos anos 1970 e os primeiros anos da década seguinte, com a perspectiva de implementação das atividades de controle da doença de Chagas, foram realizados dois amplos estudos epidemiológicos no Brasil, de soroprevalência da infecção humana e inquérito entomológico. Com base nos seus resultados foi possível precisar a área endêmica ou com risco de transmissão vetorial no país. Por amostragem em áreas selecionadas, foi ainda realizado inquérito eletrocardiográfico, que permitiu confirmar algumas diferenças regionais que vinham sendo apontadas em relação às manifestações clínicas da infecção ou a sua morbidade.

Os resultados destes estudos foram isoladamente reportados $^{1-4}$ e estão sendo também abordados em outros artigos que compõe a presente publicação. O que se busca aqui é o cotejo e a sobreposição dos seus resultados. Na prática, isso

\footnotetext{
*Departamento de Medicina Social, Faculdade de Medicina de Ribeirão Preto Universidade de São Paulo, Ribeirão Preto, SP. **Ex Diretor da Divisão Nacional de Doença de Chagas e da Divisão Nacional de Epidemiologia do Ministério da Saúde e Consultor da Organização Panamericana de Saúde, Brasília, DF.

Endereço para correspondência: Dr. Afonso Dias Costa Passos. Avenida Bandeirantes 3900, 14049-900 Ribeirão Preto, SP.

e-mail: apassos@fmrp.usp.br
}

já foi parcialmente realizado, como na delimitação da área inicialmente considerada endêmica para a doença de Chagas (Figura 1) ${ }^{5}$. Ainda que para isso se tenha tomado basicamente os dados relativos à distribuição dos vetores domiciliados, foi considerada a possibilidade de que pudesse haver alguma inconsistência em relação àqueles de soroprevalência.

O principal interesse em sobrepor-se a distribuição geográfica das principais espécies de vetor (Triatoma infestans, Panstrongylus megistus, T. brasiliensis, T. pseudomaculata e T. sordida) e a proporção de sororreagentes por município seria a confirmação da diferente importância de cada uma delas na transmissão domiciliar da doença de Chagas. Nas Figuras 2 a 6 seguintes apresenta-se o mapeamento feito a partir dos dados do inquérito triatomínico inicial $(1975 / 83)^{6}$ e dos resultados do inquérito sorológico de base $(1975 / 80)^{1}$.

É muito evidente a relação entre a(s) espécie(s) de vetor presente(s) e as taxas de soro-prevalência. Nas áreas com Triatoma infestans, o principal vetor, porque estritamente domiciliado, altamente antropofílico e com maiores taxas de infecção natural por Trypanosoma cruzi, a infecção humana foi significativamente mais frequente (Figura 2). Por mais 


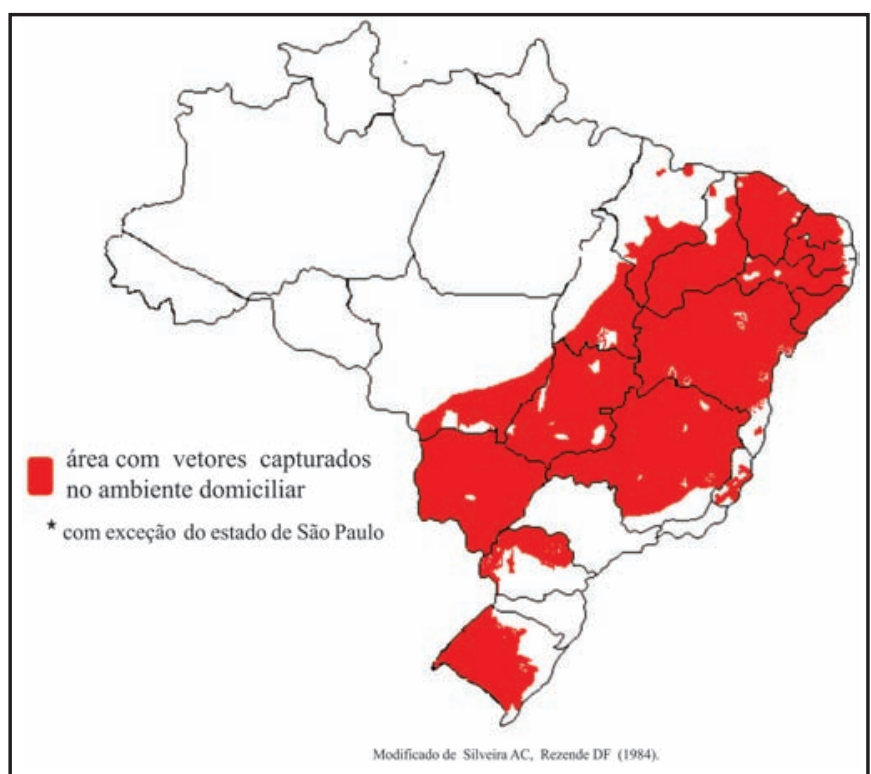

FIGURA 1 - Área originalmente endêmica para a doença de Chagas, com risco de transmissão vetorial domiciliar. Brasil*. 1983.

esperada e óbvia que pudesse ser a correlação entre estes eventos, aqui pode-se comprová-la de forma inquestionável, desde que está apoiada em dados objetivos e em grande escala. A chamada Metade Sul do Estado do Rio Grande do Sul e as regiões do Triângulo e norte de Minas Gerais, áreas em que foram registrados os maiores índices de sororreatividade para a infecção chagásica, são aquelas em que no passado se verificaram as maiores taxas de infestação por T. infestans. Em alguma medida, o mesmo aplica-se a municípios do oeste da Bahia e do extremo norte do Paraná, na divisa com São Paulo.

Além da infestação, especialmente importante, a antiguidade do ingresso de T. infestans nessas áreas, sabido desde há muito, seria outro elemento a considerar na explicação dos altos índices de soro-prevalência aí verificados. Tanto é assim que naquelas áreas há menos tempo infestadas, como em alguns estados da região nordeste (Pernambuco, Paraíba e Piauí) e do centro-oeste (Mato Grosso e Mato Grosso do Sul) ${ }^{1}$ as proporções de soropositivos foram bastante inferiores.

Em relação às demais espécies, todas autóctones e com uma clara divisão de território, P. megistus, nativo da Mata Atlântica e com grande dispersão pelo interior do país, ao longo de bosques e matas ciliares, Triatoma brasiliensis e $T$. pseudomaculata com centro de endemismo no semi-árido do nordeste e T. sordida, própria do domínio dos cerrados, devese assinalar que (Figuras 3 a 6): I) a presença de nenhuma delas está manifestamente associada com altos níveis de prevalência sorológica da infecção chagásica; II) no caso de T. sordida, pela coincidência da área de em que está disperso na região central do Brasil (incluindo os Estados de Minas Gerais, Goiás e Bahia) com aquela de T. infestans, poderia aparentar importante participação na transmissão domiciliar da doença de Chagas. Isso absolutamente não acontece em decorrência da sua conhecida ornitofilia, o que faz com que seja capturado quase que exclusivamente no ambiente peri-domiciliar, com baixas taxas de infecção por T. cruzi $i^{7}$; III) na avaliação da importância vetorial do P. megistus, aquele com mais difusa distribuição no país, importa fazer a distinção de áreas em que

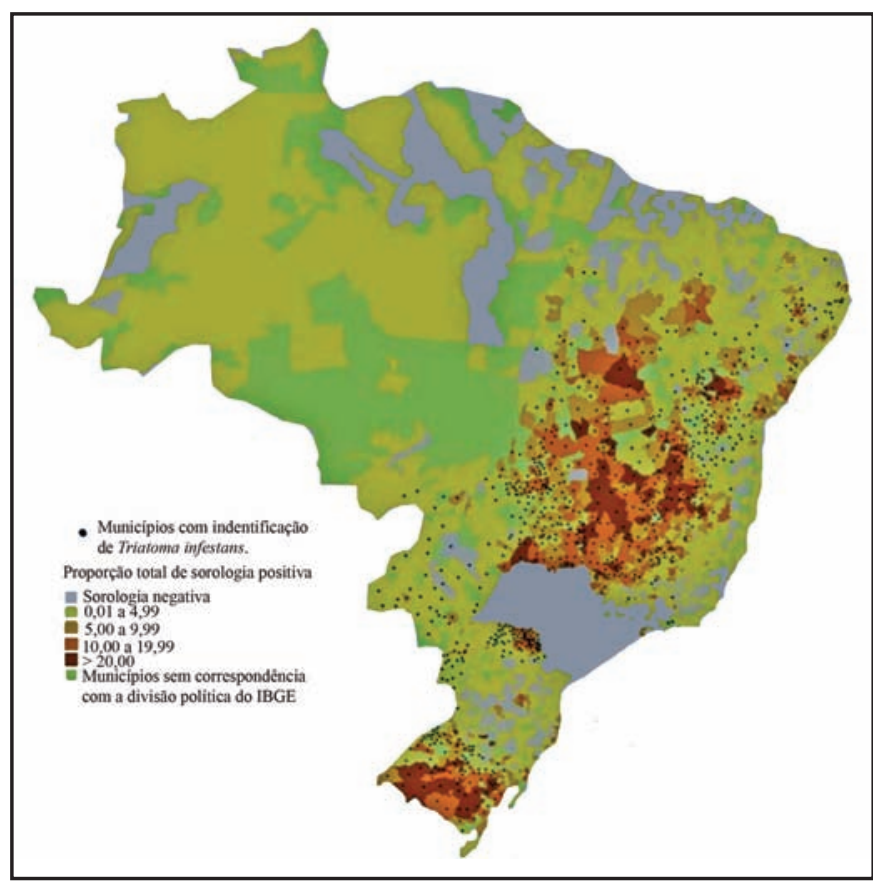

FIGURA 2 - Municípios com identificação de Triatoma infestans no Brasil*.

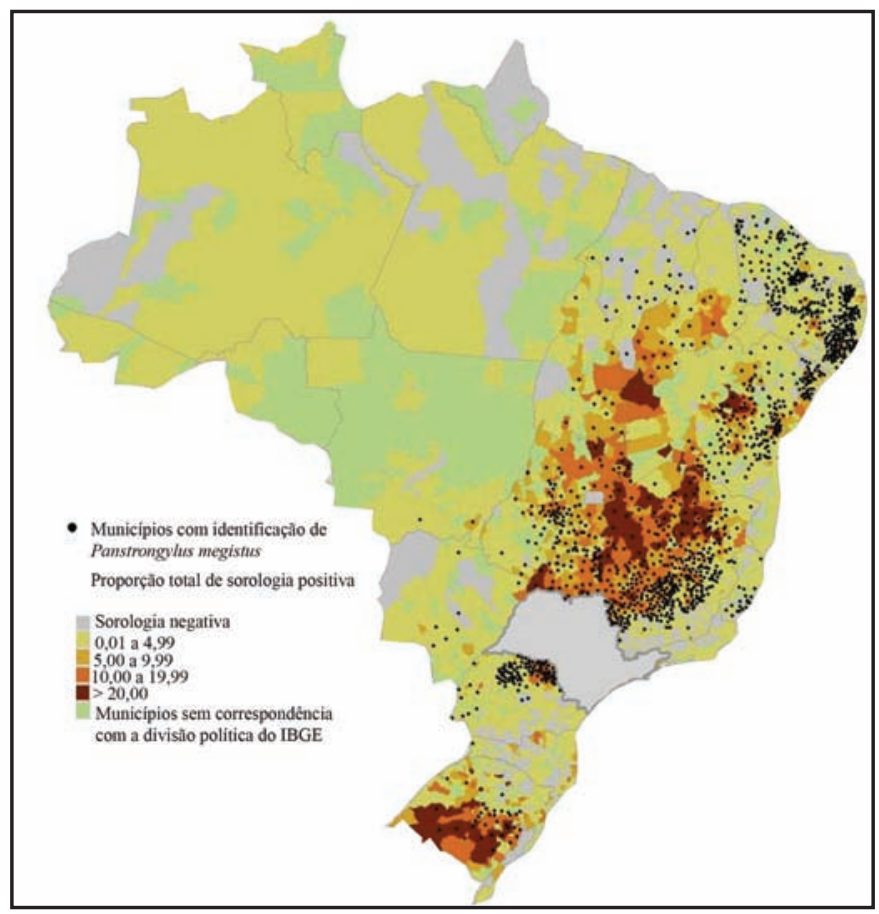

FIGURA 3 - Municípios com identificação de Panstrongylus megistus no Brasil*.

apresentava grande infestação e colonização intradomiciliar, como no litoral úmido do nordeste, e onde foram registradas taxas relativamente elevadas de infecção no inquérito de soroprevalência, daquelas em que a presença do vetor domiciliado foi, e segue sendo, rara e, frequentemente, sem colonização intradomiciliar importante, como em extensas regiões do sul do Brasil. IV) para as áreas de dispersão de T. brasiliensis e T. pseudomaculata, a infecção humana mostrada pelo inquérito de soro-prevalência foi em geral pouco expressiva, ao menos 


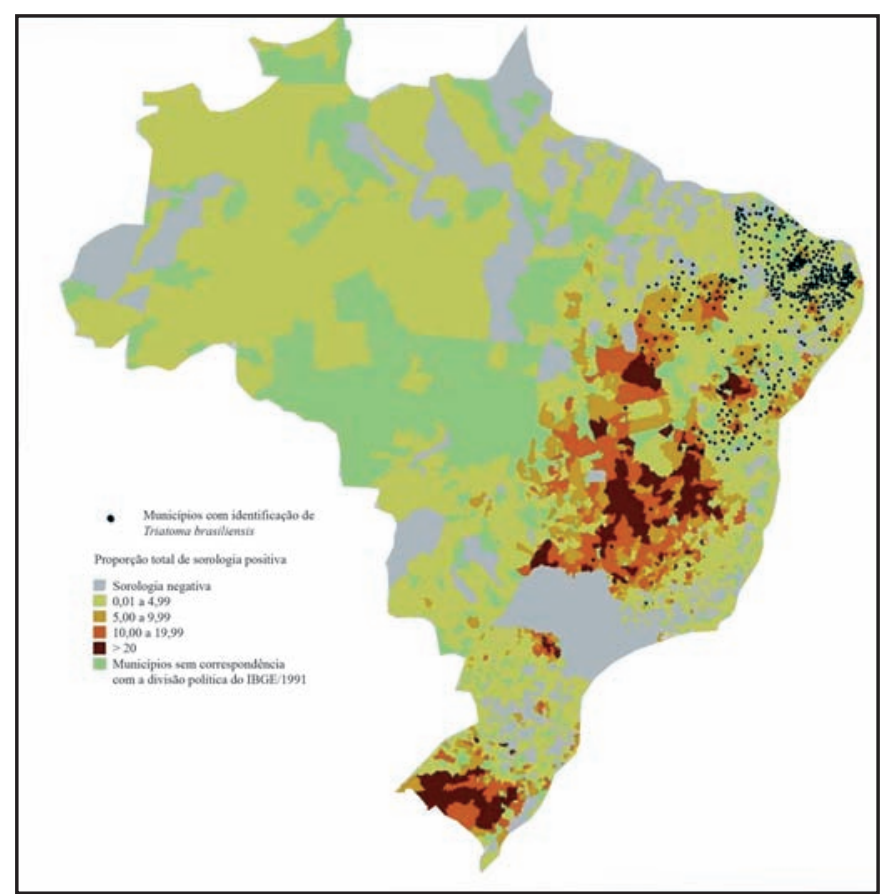

FIGURA 4 - Municípios com identificação de Triatoma brasiliensis no Brasil*.

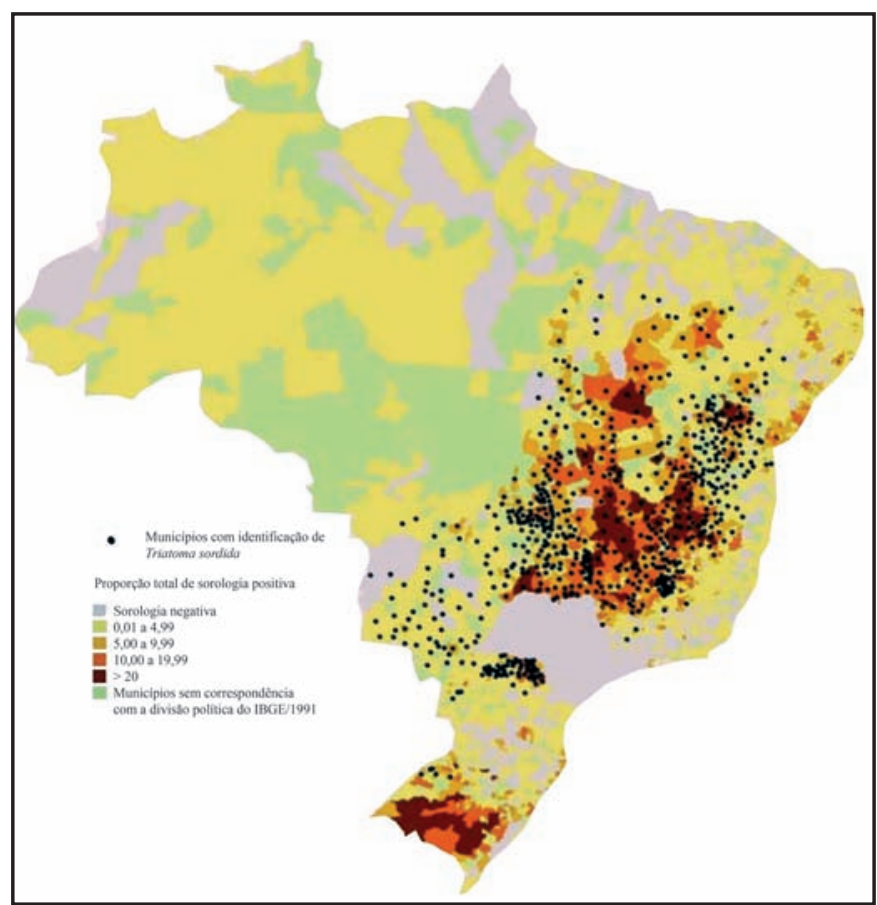

FIGURA 6 - Municípios com identificação de Triatoma sordida no Brasil*.

em relação ao que foi verificado para T. infestans e para P. megistus na zona da mata nordestina e, em especial, na região do Recôncavo Baiano.

Na Tabela 1, mostra-se o número de municípios infestados por cada uma das principais espécies de vetor, distribuídos conforme as taxas de soroprevalência conhecidas pelo Inquérito Nacional 1975/80.

Os dados reafirmam as observações feitas anteriormente, destacando-se os maiores percentuais de infestação por

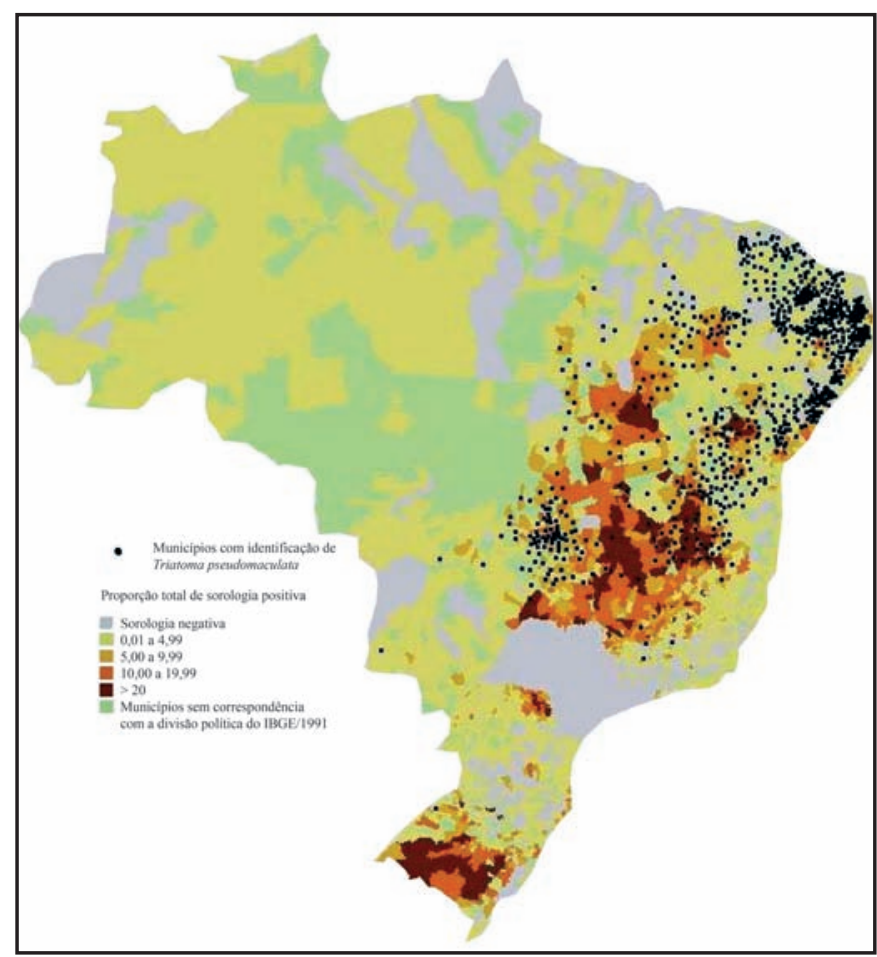

FIGURA 5 - Municípios com identificação de Triatoma pseudomaculata no Brasil*.

P. megistus, T. brasiliensis e T. pseudomaculata em áreas com reduzidas frequências de soro-positividade. Situação inversa é mostrada para T. infestans e T. sordida, cabendo lembrar que o achado para este último reflete tão somente a sua distribuição geográfica sobreposta ao primeiro, haja vista a sua pouca importância na transmissão da infecção chagásica em nosso meio.

Importa observar que a malha municipal que serviu para a construção da tabela e dos mapas aqui apresentados foi baseada no censo demográfico de 1991, do Instituto Brasileiro de Geografia e Estatística (IBGE) ${ }^{8}$. Com a criação de novos municípios, em alguns casos não foi possível estabelecer sua correspondência com os dados colhidos nos inquéritos nacionais, triatomínico ou sorológico.

Com base nos dados colhidos no Inquérito Nacional de Soro-prevalência, realizou-se, entre 1977 e 1981, um Inquérito Eletrocardiográfico em onze estados selecionados. Entre eles, aqueles com maiores taxas de sororreagentes, pela ordem Rio Grande do Sul (8,8\%), Minas Gerais (8,8\%), Goiás (7,4\%), Sergipe (6\%), Bahia (5,4\%), Piauí (4\%), Paraná (4\%), Paraíba $(3,5 \%)$, Pernambuco $(2,8 \%)$ e Alagoas $(2,5 \%)$. Foi incluído também o Ceará, estado em que a prevalência estimada $(0,8 \%)$ esteve bastante abaixo do que era a princípio esperado.

Os resultados inicialmente publicados mostraram que a frequência de alterações eletrocardiográficas entre os sororreagentes foi maior nos Estados de Goiás $(55,6 \%)$, Minas Gerais (53,5\%), Piauí (45\%), Bahia (44,3\%) e Paraná (34,5\%); e que nos Estados de Sergipe, Alagoas, Rio Grande do Sul e Ceará não houve diferença significante de alterações eletrocardiográficas, entre soro reagentes e não reagentes ${ }^{4}$.

Esses achados corroboram a existência de diferenças regionais importantes em relação às manifestações clínicas da 
TABELA 1 - Número e percentual de municípios infestados pelas principais espécies vetoras da doença de Chagas distribuídos segundo os resultados do Inquérito sorológico de prevalência da infecção chagásica no Brasil, 1975/1980 (conforme intervalos de classe arbitrados).

\begin{tabular}{|c|c|c|c|c|c|c|c|c|c|c|}
\hline \multirow[b]{3}{*}{ Proporção de amostras (\%) } & \multicolumn{10}{|c|}{ Municípios infestados por espécie } \\
\hline & \multicolumn{2}{|c|}{$\begin{array}{l}\text { Triatoma } \\
\text { infestans }\end{array}$} & \multicolumn{2}{|c|}{$\begin{array}{c}\text { Panstrongylus } \\
\text { megistus }\end{array}$} & \multicolumn{2}{|c|}{$\begin{array}{l}\text { Triatoma } \\
\text { brasiliensis }\end{array}$} & \multicolumn{2}{|c|}{$\begin{array}{c}\text { Triatoma } \\
\text { pseudomaculata }\end{array}$} & \multicolumn{2}{|c|}{$\begin{array}{l}\text { Triatoma } \\
\text { sordida }\end{array}$} \\
\hline & $\mathrm{n}^{\circ}$ & $\% *$ & $\mathrm{n}^{\circ}$ & $\%^{*}$ & $\mathrm{n}^{\circ}$ & $\%^{*}$ & $\mathrm{n}^{\circ}$ & \%* & $\mathrm{n}^{\circ}$ & $\%^{*}$ \\
\hline 0,01 a 4,99 & 259 & 36,8 & 631 & 49,5 & 328 & 78,4 & 484 & 65,0 & 241 & 37,7 \\
\hline 5,0 a 9,99 & 148 & 21,1 & 222 & 17,4 & 52 & 12,4 & 125 & 16,7 & 141 & 22,0 \\
\hline 10,0 a 19,99 & 175 & 24,9 & 212 & 16,6 & 28 & 6,7 & 90 & 12,0 & 156 & 24,4 \\
\hline 20,0 e mais & 100 & 14,2 & 105 & 8,2 & 5 & 1,2 & 31 & 4,1 & 81 & 12,7 \\
\hline Sorologia negativa & 21 & 3,0 & 93 & 7,3 & 5 & 1,2 & 17 & 2,3 & 20 & 3,1 \\
\hline Sem informação ${ }^{* *}$ & 23 & & 26 & & 66 & & 93 & & 21 & \\
\hline$\overline{\text { Total }}$ & 726 & & 1.299 & & 484 & & 840 & & 660 & \\
\hline
\end{tabular}

*considerados os municípios com informação válida, ** municípios em que não foi possível estabelecer correspondência com a divisão política IBGE 1991.

doença de Chagas no país ${ }^{9,10}$. Os resultados dos dois inquéritos (sorológico e eletrocardiográfico) foi especialmente destoante no caso do Rio Grande do Sul, estado que apresentou a mais elevada proporção de positivos no inquérito sorológico e onde alterações eletrocardiográficas e clínicas relevantes fizeram-se presentes em valores reduzidos. Este fato tem sido associado às variações de linhagens de Trypanosoma cruzi nas diferentes regiões do Brasil, resultando na diversidade de manifestações clínicas observadas entre elas.

\section{REFERÊNCIAS}

1. Camargo ME, Silva GR, Castilho EA, Silveira AC. Inquérito sorológico da prevalência de infecção chagásica no Brasil, 1975/1980. Rev Inst Med Trop São Paulo 1984; 26:192-204.

2. Forattini OP, Barata JMS, Santos JLF, Silveira AC. Hábitos alimentares, infecção natural e distribuição de triatomíneos domiciliados na região Nordeste do Brasil. Rev Saude Publica São Paulo 1981; 15:113-164.

3. Forattini OP, Barata JMS, Santos JLS, Silveira AC. Hábitos alimentares, infecção natural e distribuição de triatomíneos domiciliados na região central do Brasil. Rev Saude Publica São Pau1o 1982; 6:171-204.

4. Macêdo VO, Prata A, Silva GR. Prevalência de alterações eletrocardiográficas em chagásicos. (Informações preliminares sobre o inquérito eletrocardiográfico nacional). Arq Bras Cardiol 1982; 38:261-264.

5. Silveira AC, Souza PC, Silveira Neto HV. Importância de espécies secundárias de triatomíneos na transmissão domiciliar da doença de Chagas no Triângulo Mineiro e Alto Paranaíba. Rev Soc Bras Med Trop 1993; 26 (supl I): 200.

6. Silveira AC, Feitosa VR, Borges R. Distribuição de triatomíneos capturados no ambiente domiciliar, no período de 1975-83, Brasil. Rev Bras Malariol D Trop 1984; 36:15-312.

7. Diotaiuti L. Importância atual e perspectivas de controle do Triatoma sordida em Minas Gerais [tese de doutorado]. [Belo Horizonte]: Universidade Federal de Minas Gerais; 1991.

8. Instituto Brasileiro de Geografia e Estatística. Censo Demográfico de 1991. Rio de Janeiro; 1994.

9. Coura JR. Reunião sobre diferenças geográficas na doença de Chagas. Brasília; 1975.

10. Silveira AC, Rezende DF. Epidemiologia e controle da transmissão vetorial da doença de Chagas no Brasil. Rev Soc Bras Med Trop 1994; 27:11-22. 